\title{
The role of line junctions in object recognition: The case of reading musical notation
}

\author{
Yetta Kwailing Wong ${ }^{1}$ • Alan C.-N. Wong ${ }^{2}$
}

Published online: 30 April 2018

(C) Psychonomic Society, Inc. 2018

\begin{abstract}
Previous work has shown that line junctions are informative features for visual perception of objects, letters, and words. However, the sources of such sensitivity and their generalizability to other object categories are largely unclear. We addressed these questions by studying perceptual expertise in reading musical notation, a domain in which individuals with different levels of expertise are readily available. We observed that removing line junctions created by the contact between musical notes and staff lines selectively impaired recognition performance in experts and intermediate readers, but not in novices. The degree of performance impairment was predicted by individual fluency in reading musical notation. Our findings suggest that line junctions provide diagnostic information about object identity across various categories, including musical notation. However, human sensitivity to line junctions does not readily transfer from familiar to unfamiliar object categories, and has to be acquired through perceptual experience with the specific objects.
\end{abstract}

Keywords Perceptual expertise $\cdot$ Reading $\cdot$ Music reading $\cdot$ Visual feature

Visual features are fundamental to the understanding of object recognition. Computational models of object recognition typically begin with a stage of visual-feature processing (Mccleery et al., 2008; McClelland \& Rumelhart, 1981; Riesenhuber \& Poggio, 1999; Testolin, Stoianov, \& Zorzi, 2017; Tong, Joyce, \& Cottrell, 2008). However, researchers have not agreed on the exact characterization of the visual features. Instead, they adopted different types of visual features in different theories and models, including edges (Riesenhuber \& Poggio, 1999), Gabor filters (Mccleery et al., 2008; Tong et al., 2008), and three-dimensional shape primitives called geons (Biederman, 1987). Others suggest that informative features are immediately complex and vary depending on the specific object category (Ullman, 2007).

Yetta Kwailing Wong yetta.wong@cuhk.edu.hk

Alan C.-N. Wong alanwong@cuhk.edu.hk

1 Department of Educational Psychology, The Chinese University of Hong Kong, Rm 308, Ho Tim Building, Shatin, NT, Hong Kong

2 Department of Psychology, The Chinese University of Hong Kong, 344 Sino Building, Shatin, NT, Hong Kong
Other properties are also important for discriminating between objects within a particular category, such as small local features that are not informative of the object category per se (Epshtein \& Ullman, 2006), the overall complexity of the object pattern (Pelli, Burns, Farell, \& Moore-Page, 2006), and the spatial arrangements of parts (Maurer, Grand, \& Mondloch, 2002; Yin, 1969). Moreover, it has been proposed that informative features can sometimes be effectively recovered by simple perceptron networks without hidden layers (e.g., in the case of letter identification; Hannagan \& Grainger, 2013).

Among the proposed candidate features important for object recognition, line junctions seem to be a common informative feature for real-world objects. Line junctions indicate where edges or lines meet, and are also referred to as vertices, $\mathrm{T}$ or L junctions, or line coterminations (Biederman, 1987). Removing vertices is more detrimental than removing midsegments of lines for recognizing common objects (Biederman, 1987), as well as letters and words (Lanthier, Risko, Stolz, \& Besner, 2009; Szwed, Cohen, Qiao, \& Dehaene, 2009). Line terminations, which also signal the end of a line segment similar to vertices, are also found to be particularly important for human letter recognition (Fiset et al., 2008). While sensitivity to vertices is common to human and nonhuman mammalian vision, such as pigeons (Gibson, 
Lazareva, Gosselin, Schyns, \& Wasserman, 2007), it is also where the human visual system and computer models diverge - many computer models do not generally find vertices particularly informative compared with other features (Fiset et al., 2008; Gibson et al., 2007).

Some important questions regarding the role of line junctions in object recognition remain unclear. For example, is sensitivity to line junctions general to different categories other than common objects and words? How do humans develop sensitivity to line junctions? Specifically, is our visual system naturally tuned to line junctions, or is it learned? In this article, we addressed these questions in a relatively understudied domain of visual perceptual expertise - the reading of musical notation.

Musicians are often highly fluent in recognizing musical notation and are about three times faster than music-reading novices in recognizing four-to-five-note sequences (Wong \& Gauthier, 2010b, 2012). Music-reading experts experience less difficulty in discriminating notes in crowded images (Wong \& Gauthier, 2012; Wong \& Wong, 2016). Interestingly, though, when responding to a particular note, they find it difficult to disregard the neighboring notes completely (Wong \& Gauthier, 2010b). Studying musicreading expertise helps us to understand human sensitivity to line junctions for several reasons. First, for musical notes, the line junctions between the dot and the staff lines inform the pitches ${ }^{1}$ of adjacent notes. Specifically, if the note is on one of the staff lines, the junctions are convex on both sides of the dot, whereas if the note is between two staff lines, the junctions are three concave vertices and a roughly $90^{\circ}$ vertex (see Fig. 1). Given these differences, the type and number of the line junctions inform the exact position of the dots, which in turn facilitate the identification of notes among spatially adjacent alternatives. Second, there is a wide range of visual skills in reading musical notation in the population. This allows one to address the development of the sensitivity to line junctions in note recognition, and the relationship between sensitivity to line junctions and skill levels in music reading. This is relatively difficult to achieve in other domains, such as word reading and face recognition, in which skill levels tend to appear at the two ends of the continuum.

In this article, we tested the importance of line junctions in reading musical notation. Participants judged whether two sequentially presented note sequences were identical or different. The line junctions of the notes were either intact or removed. If line junctions are informative visual features to which human visual systems are sensitive by default, then removing them should impair participants' recognition

\footnotetext{
${ }^{1}$ In the Western musical notational system, pitch refers to the frequency of the tone denoted by each musical note. It is mainly indicated by the position of the musical note on the five-line staff, with small modifications indicated by other symbols on the music score (e.g., accidentals such as \# orb).
}

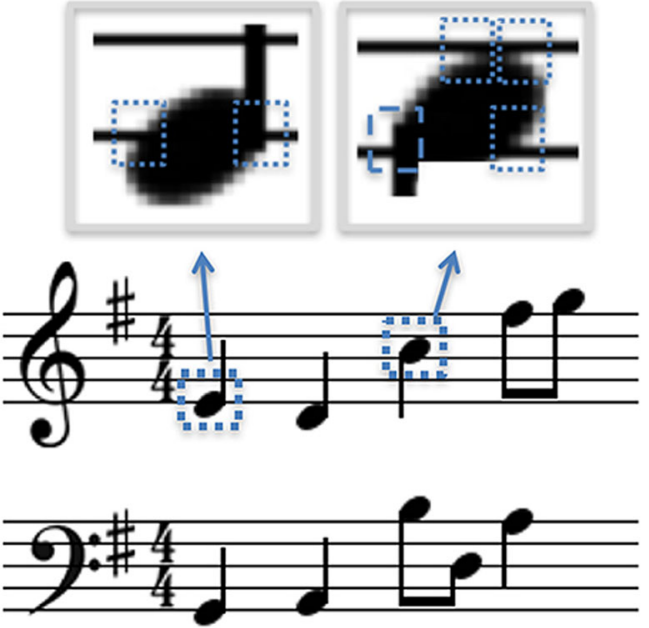

Fig. 1 a The line junctions of a music sequence. For notes on a line, there are two convex junctions on the left and right side of the dot (top left panel). For notes in the space between lines, there are three concave junctions at both top corners and the bottom right corner of the dot, and a roughly $90^{\circ}$ junction at the bottom left corner (top right panel). Note that the exact positions of these concave and $90^{\circ}$ junctions depend on the pointing direction of the notes

regardless of skill levels in reading musical notation. If line junctions were critical features that musicians learn to pick up during development of reading skills, then they should selectively impair expert performance and affect novices to a lesser extent (if any). Importantly, if the line junctions become more perceptually important for better experts, then the degree of performance impairment by removing line junctions should be predicted by individual fluency in music reading. To preview our results, we observed that removal of line junctions of the notes selectively affected experts' performance, and it impaired performance more for faster music readers.

\section{Method}

\section{Participants}

We aimed at recruiting three groups, comprising expert, intermediate, and novice readers, each including about 22 participants after data exclusion (e.g., with outlier or near-chance performance; see below). The power estimation was performed using $\mathrm{G}^{*}$ Power 3.1. Assuming a medium effect size $\left(f^{2}=0.2\right)$, this sample size would allow the detection of any effect at $\alpha=0.05$, with a power of 0.8 for a repeated-measures ANOVA with a three-level between-subjects factor and a twolevel within-subjects factor.

Seventy-six participants were recruited in this study. Fortyfour experts were recruited as music readers and were split into the expert and intermediate reader groups based on their fluency in reading musical notation (see below; Table 1). Twenty-three participants who reported that they could not 
Table 1 Descriptive statistics and perceptual fluency of the participants

\begin{tabular}{llllll}
\hline Group & Age $(S D ;$ range $)$ & $N(\mathrm{M} / \mathrm{F})$ & Years $(S D)$ & Note fluency $(S D)$ & Digit fluency $(S D)$ \\
\hline Expert & 21.4 & $22(4 / 18)$ & $14.5(2.94)$ & $429(95)$ & $254(115)$ \\
Intermediate & $(2.87 ; 18-30)$ & $22(4 / 18)$ & $13.6(4.60)$ & $830(169)$ & $335(156)$ \\
Novice & 22.0 & $23(3 / 20)$ & $1.02(1.42)$ & $944(227)$ & $300(157)$ \\
& $(3.76 ; 18-35)$ & & & \\
\hline
\end{tabular}

Note. $N=$ number of participants in each group. Years = average number of years of music reading training for each group. Note fluency and digit fluency $=$ presentation duration threshold of perceiving notes and digits, respectively, as measured in the perceptual fluency task in the nearest millisecond

read music were recruited as novices. ${ }^{2}$ Data of three additional music readers and six additional novices were excluded from data analyses because of outlier performance in the perceptual fluency task (i.e., with a duration threshold more than three standard deviations from the mean of the rest of the group with either stimulus category). All reported normal or corrected-tonormal vision and gave informed consent according to the guidelines of the Institutional Review Board of City University of Hong Kong and the Chinese University of Hong Kong.

\section{Stimuli and design}

The experiment was conducted using MATLAB (Natick, MA) with the Psychophysics Toolbox extension (Brainard, 1997; Pelli, 1997). Participants were first introduced to a pitch sequential matching task in which the effect of removing line junctions was measured. Then they took a perceptual fluency test in which their individual music reading ability was assessed.

In the sequential matching task, 128 four-note sequences were randomly generated with MATLAB under the constraints that (a) the pitch of each note ranged from D4 to G5, and (b) the pitches of the four notes were different within a sequence. Half were normal sequences and the other half had the line junctions removed. All stimuli were black on a white background and subtended a visual angle of about $5.8^{\circ} \times 3.9^{\circ}$ with a viewing distance of about $60 \mathrm{~cm}$. During each trial, a fixation appeared for $500 \mathrm{~ms}$, followed by the first sequence for $600 \mathrm{~ms}$ (see Fig. 2). After a 500-ms mask, the second sequence appeared. Participants judged if the two sequences were identical in pitch by pressing a key as quickly and accurately as possible. The mask was a grayscale image of waterdrop patterns used in previous studies on expert object recognition (Wong \& Gauthier, 2010b; Wong, Twedt, Sheinberg, \& Gauthier, 2010). Participants were required to

\footnotetext{
2 The information of the music training background of one music reader and two novices were lost due to technical difficulty. However, their music-reading fluency was consistent with the range of performance of their group, and the result patterns were not affected by removing these participants from the analyses.
}

respond within 3,000 $\mathrm{ms}$, and the absence of a response was taken as incorrect. For the "different" trials, one of the four notes in the second sequence would be shifted by one step such that the two sequences would be highly similar. The position of the shifted note and the direction of the shift were counterbalanced across trials. There were 10 practice trials with feedback, followed by the experimental trials without feedback. The dependent measures were sensitivity A' and response time for correct responses (RT). The 128 experimental trials consisted of 64 trials for each condition (normal vs. junctions removed). Trials in different conditions were randomized and divided into two blocks. The number of trials in which no response was recorded within the 3-s response window was $0.37 \%$ and $0.58 \%$ for the normal and the junctions removed conditions, respectively (or 16 and 25 trials, respectively, out of 4,288 trials in all participants).

In the perceptual fluency test, individual fluency in music reading was quantified. During each trial, a central fixation was shown for $200 \mathrm{~ms}$, followed by a premask for $500 \mathrm{~ms}$ and a target stimulus for a varied duration. After a postmask for $500 \mathrm{~ms}$, the target stimulus and a highly similar distractor were shown side by side, and participants were asked to select the target stimulus by key press without time limit. A total of 400 four-note sequences were generated separately under constraints similar to the main experiment. The contrast of the stimuli was reduced by about $60 \%$ to prevent the ceiling effect. The stimuli subtended a visual angle of about $6.8^{\circ} \times 3.9^{\circ}$ with a viewing distance of about $60 \mathrm{~cm}$. The mask was created by fragments of pseudoletters used in a previous study (Gauthier, Wong, Hayward, \& Cheung, 2006). The presentation duration of the target stimulus was determined by a 3down-1-up staircase procedure to keep participants at $80 \%$ accuracy (García-Pérez, 1998). For each estimate, the initial presentation time was $700 \mathrm{~ms}$. The presentation duration was reduced by $30 \%$ following three consecutive correct responses, and increased by $35 \%$ following one incorrect response. The count of correct responses was reset to zero after each duration adjustment. The lower boundary of the presentation duration was set to $40 \mathrm{~ms}$ to prevent showing undetectable stimuli (García-Pérez, 1998). For achieving stable estimates, the staircase would stop when both of the following 


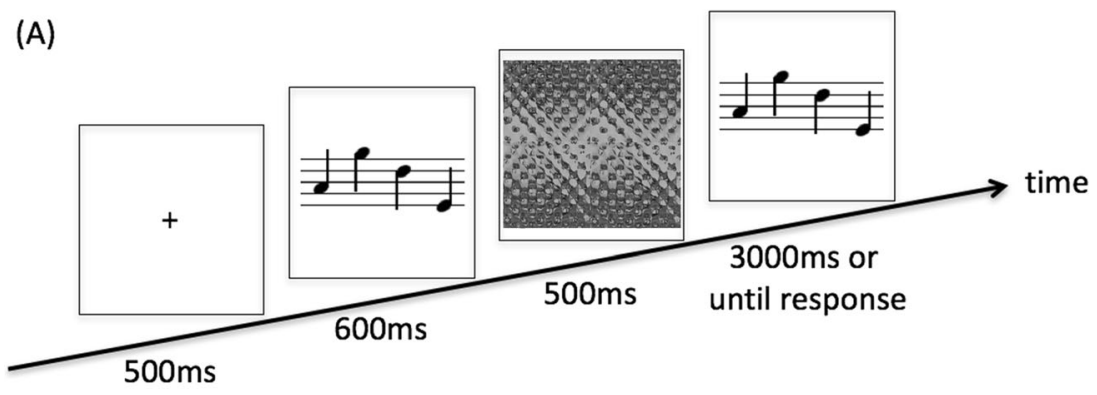

(B)

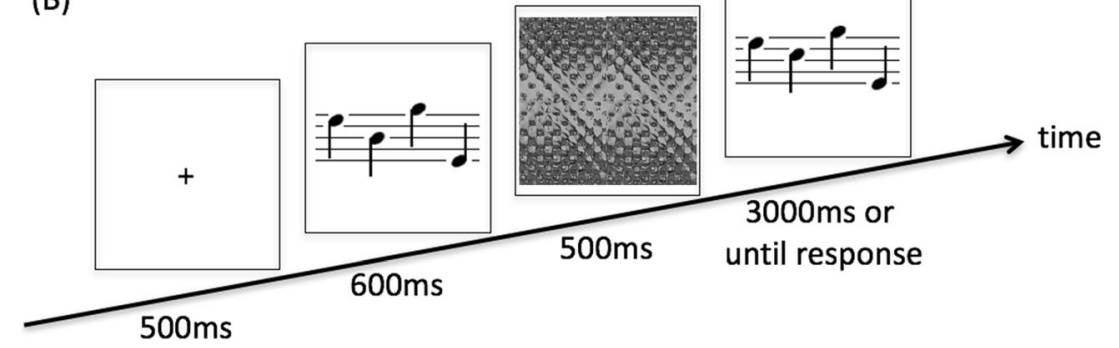

Fig. 2 The design of the sequential matching task. a A "same" trial in which the pitches of all notes were identical in the two sequences. b A "different" trial with the line junctions of the notes removed in both

criteria were met: (a) there were 10 small-magnitude reversals of the direction of duration adjustment (i.e., only reversals preceded by four or less consecutive adjustments in the same direction were counted into the 10 reversals), and (b) the last five reversals were consecutive smallmagnitude reversals. The number of reversals was determined by pilot testing which showed that most participants reached plateau performance at about 10 reversals. The duration threshold was the average duration of all reversals. Five practice trials were included before the experimental trials. Individual fluency in music reading was determined as the average duration threshold from two staircase estimates. Apart from musical notes, the presentation duration threshold of six-digit strings, a control category, was also estimated with a similar procedure. The threshold for digits offered a measure of the general perceptual abilities that were not specifically tied to music reading. Six digits were used instead of four because of the ceiling effect observed in pilot testing. The digit strings subtended a similar visual angle as that of the music sequences.

\section{Results}

\section{Perceptual fluency for notes and digits}

To confirm the group differences in music reading expertise, a $3 \times 2$ ANOVA, with group (expert/intermediate/novice) and stimulus category (notes/digits) was performed on duration threshold in the perceptual fluency task. We observed a main images. It was a "different" trial because the second note on the second image was different from that in the first image

effect of group, $F(2,64)=42.3, p=.0001, \eta_{\mathrm{p}}^{2}=0.57$, and a main effect of stimulus category, $F(1,64)=251.2, p=.0001$, $\eta_{\mathrm{p}}{ }^{2}=0.80$. Importantly, a significant group $\times$ stimulus category interaction was observed, $F(2,64)=25.9, p=.0001, \eta_{\mathrm{p}}^{2}$ $=0.45$. A post hoc Scheffé test $(p<.05)$ revealed that experts recognized the music sequences more fluently than did intermediates, who were marginally more fluent than novices ( $p=$ .068; see Table 1). The three groups had similar fluency in recognizing digit strings (see Table 1). These results confirmed that experts had a higher level of music reading expertise than other groups, which could not be explained by general perceptual abilities as measured with digit strings.

\section{Sequential matching task}

The removal of line junctions of the notes selectively impaired performance of the music readers on both $\mathrm{A}^{\prime}$ and correct RT (see Fig. 3). A $3 \times 2$ ANOVA with group (expert/intermediate/ novice) and line junction (present/absent) on A' revealed a main effect of group, $F(2,64)=4.57, p=.014, \eta_{p}{ }^{2}=0.12$, with the experts performing better than the novices, but similarly to the intermediates (post hoc LSD test, $p<.05$ ). The main effect of line junction was significant, $F(1,64)=44.4, p$ $=.0001, \eta_{\mathrm{p}}{ }^{2}=0.41$, with a higher sensitivity when line junctions of the notes were present rather than absent. Importantly, the group $\times$ line junction interaction was significant, $F(2,64)$ $=5.70, p=.0053, \eta_{\mathrm{p}}{ }^{2}=0.082$ (see Fig. 3 and Table 2). A post hoc Scheffé test $(p<.05)$ showed that the A' was higher when the line junctions were present than absent for both the experts 
(A)

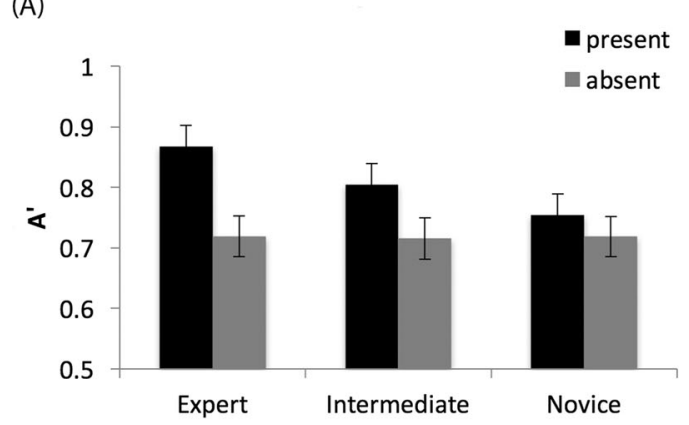

(B)

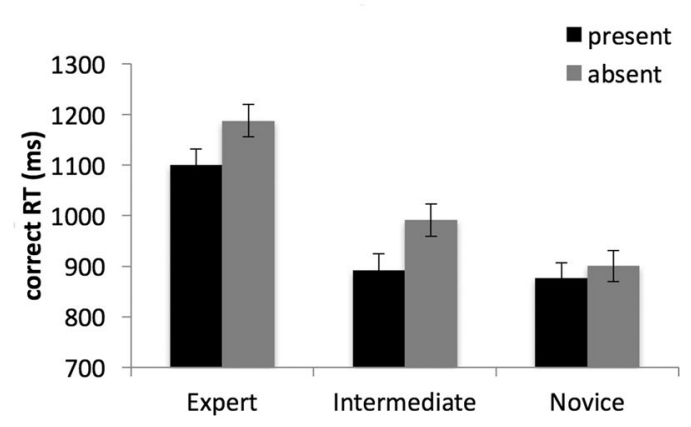

(C)

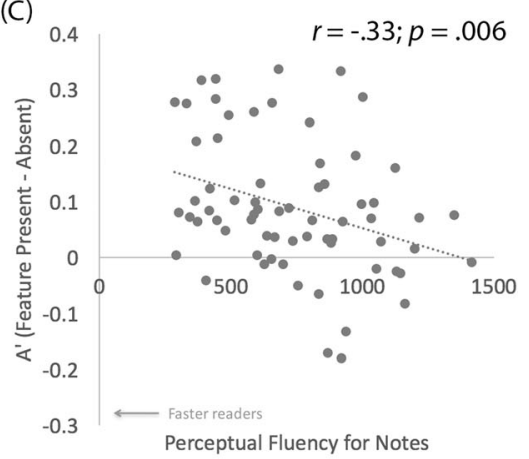

(D)

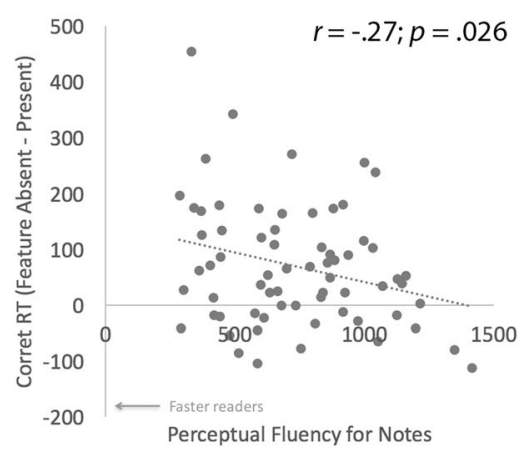

Fig. 3 Bar charts show the performance for the three groups of music readers when the line junctions were present or absent in $\mathrm{A}^{\prime}$ (a) and in correct response time (b). The error bars plot the $95 \%$ confidence intervals of the Group $\times$ Line Junction interaction. The scatterplots

and the intermediates, but not for the novices. The A' was similar for all groups when the line junctions were absent.

For correct RT, a similar ANOVA revealed a main effect of group, $F(2,64)=8.14, p=.0007, \eta_{\mathrm{p}}{ }^{2}=0.20$, with the experts responding more slowly than the other two groups (post hoc LSD test, $p<.05$ ). The main effect of line junction was significant, $F(1,64)=29.6, p=.0001, \eta_{\mathrm{p}}{ }^{2}=0.32$, with a faster response when the line junctions were present than when the line junctions were absent. Importantly, the group $\times$ line junction interaction was significant, $F(2,64)=3.32, p=$

Table 2 Proportion of hit trials and false alarm (FA) trials contributing to the calculation of A', and the mean correct response time (RT) for each condition

\begin{tabular}{lllll}
\hline & Hit rate & FA rate & A $^{\prime}$ & RT (ms) \\
\hline $\begin{array}{lllll}\text { Expert } \\
\text { Present }\end{array}$ & 0.93 & 0.38 & 0.87 & 1,188 \\
Absent & 0.75 & 0.48 & 0.72 & 1,101 \\
Intermediate & & & & \\
$\quad$ Present & 0.90 & 0.52 & 0.80 & 992 \\
Absent & 0.77 & 0.51 & 0.72 & 892 \\
Novice & & & & \\
Present & 0.86 & 0.57 & 0.75 & 900 \\
Absent & 0.83 & 0.58 & 0.72 & 876 \\
\hline
\end{tabular}

show the correlation between the performance impairment created by the removal of the line junctions and the perceptual fluency of music reading in $\mathrm{A}^{\prime}$ (c) and that in correct RT (d)

$.043, \eta_{\mathrm{p}}{ }^{2}=0.094$ (see Fig. 3). A post hoc Scheffé test ( $p<$ .05 ) showed that the experts and intermediates responded more slowly when line junctions were absent than when the line junctions were present, while for the novices the correct RT was similar regardless of the presence of the line junctions. It is counterintuitive that experts responded more slowly (though more accurate) than novices in general. At least two possible factors may have contributed to this finding. One is the speed-accuracy trade-off, in which experts might have emphasized more accuracy and less speed relative to novices. The other factor is that experts could have automatically engaged in extra multimodal processes that slowed them down in a visual judgment task.

The result patterns were similar after controlling for general perceptual ability of the individuals. ANCOVAs were performed by adding the duration threshold of digit strings as a covariate to the group $\times$ line junction ANOVA above on A' and correct RT. For both ANCOVAs, the result patterns and significance levels stayed largely similar. For A', the ANCOVA revealed a main effect of group, $F(2,63)=4.07$, $p=.022, \eta_{\mathrm{p}}{ }^{2}=0.11$; a main effect of line junction, $F(1,63)=$ $7.88, p=.007, \eta_{\mathrm{p}}{ }^{2}=0.11$; and a significant group $\times$ line Junction interaction, $F(2,63)=5.52, p=.006, \eta_{\mathrm{p}}{ }^{2}=0.15$. A similar ANCOVA on correct RT revealed a main effect of group, $F(2,63)=7.09, p=.002, \eta_{\mathrm{p}}{ }^{2}=0.18$; a main effect of line junction, $F(1,63)=11.2, p=.001, \eta_{\mathrm{p}}{ }^{2}=0.15$; and a 
significant group $\times$ line junction interaction, $F(2,63)=3.38, p$ $=.040, \eta_{\mathrm{p}}{ }^{2}=0.097$. These results suggest that individual difference in general perceptual abilities cannot explain the selective impairment of pitch recognition performance of experts and intermediates by the removal of the line junctions.

Correlation analyses showed that individual music reading fluency predicts the degree of performance impairment caused by removing the line junctions (see Fig. 3). For A', performance impairment (defined as [the present $A^{\prime}-$ the absent $\mathrm{A}^{\prime}$ ]) was correlated with the duration threshold of note sequences, $r=-.33, p=.006$. A multiple-regression analysis, with both the duration threshold of note sequences and that of digit strings as predictors, showed that performance impairment was predicted by the duration threshold of note sequences $(\beta=-.00014, S E=.0001, t=-2.78, p=.0071$; see Fig. 3c), but not by that of digit strings $(\beta=-.00000192, S E=$ $.0001, t=-0.019, p=.985)$. For correct RT, performance impairment (defined as [the absent RT - the present RT]) was correlated with the duration threshold of note sequences, $r=-.27, p=.026$. A similar multiple regression analysis showed that performance impairment was predicted by the duration threshold of note sequences $(\beta=-0.10, S E=.047$, $t=-2.14, p=.036$; see Fig. $3 \mathrm{~d}$ ), but not by that of digit strings $(\beta=-.069, S E=.091, t=-0.75, p=.45)$.

In sum, the removal of line junctions of the notes selectively impaired performance of the music readers on both $A^{\prime}$ and correct RT. Importantly, the more fluent one read music sequences, the more impaired one was once the line junctions of the notes were removed, indicated by both A' and correct RT. These effects cannot be explained by individual differences in general perceptual abilities, because the results held after accounting for the contribution of the fluency with digit strings in both the ANCOVA and multiple regression analyses.

\section{Discussion}

Using a sequential matching task, we tested the importance of line junctions as visual features in musical notation reading. We observed that removing line junctions of the notes selectively damaged the pitch recognition performance of experts and intermediates, but not that of novices. Removing line junctions caused larger performance impairment in more fluent music readers.

The implications of the current findings are twofold. First, line junctions are informative features not only for recognizing common objects, letters, and words (Biederman, 1987; Fiset et al., 2008; Gibson et al., 2007; Lanthier et al., 2009; Szwed et al., 2009) but also for recognizing musical notation. Line junctions carry information about the relationship of different lines or features, which is harder to infer or recover compared with the removal of a single feature (e.g., midsegment; Lanthier et al., 2009). This is understandably helpful for identifying common objects, letters, and words and may be particularly helpful in fluent music reading, in which readers aim to recognize the pitches of multiple notes in small sizes with a glance. Detecting the exact spatial positions of the multiple dots means catching the relationship between multiple tiny dots and the five-line staff, which could be visually challenging. Learning to discriminate the number and type of line junctions may help, which may have driven experts to rely on them more heavily in more fluent music reading. This may also explain why human preference to line junctions is not seen in ideal observers' models (Fiset et al., 2008; Gibson et al., 2007), since these models attempt to recognize single letters, which do not include the task demands of figuring out multiple letters or words and their relationships quickly and with high acuity.

The second implication of the current study is that, despite line junctions being a common diagnostic feature for various categories, sensitivity to line junctions is not an ability that the human visual system demonstrates readily for all objects. Instead, it has to be acquired for the specific object category concerned. The music reading novices in the current study were presumably equipped with real-life knowledge and experience about the usefulness of line junctions in common objects, letters, and words. Yet they failed to use line junctions to inform perceptual decisions when musical notes were concerned. This suggests that the knowledge and the sensitivity of the visual cortex to these line junctions (e.g., Szwed et al., 2011) do not readily transfer from familiar objects to unfamiliar objects. Instead, such sensitivity develops as one acquires perceptual expertise with a specific category of objects. In the current study, the reliance of expert music readers on line junctions was likely developed as a result of extensive experience developed with musical notation. This in turn may have supported fluent music reading, as suggested by the observation that sensitivity to line junctions predicts one's fluency in reading musical notation. The findings offer an explanation to the observed perceptual advantage that experts have in a reduced crowding of notes (Wong \& Gauthier, 2012; Wong \& Wong, 2016) - experts may have learned to quickly detect the line junctions that inform the pitch of the notes despite the crowdedness of the image (see also Fiset et al., 2009).

The expert sensitivity to vertices may explain the unique observation that music reading expertise engages both the higher and primary visual areas (Wong \& Gauthier, 2010a; Wong, Peng, Fratus, Woodman, \& Gauthier, 2014). While expert object processing typically involves the higher visual cortex (Cohen et al., 2000; Downing, Jiang, Shuman, \& Kanwisher, 2001; Epstein \& Kanwisher, 1998; James \& James, 2005; Kanwisher, McDermott, \& Chun, 1997), music reading expertise also recruits the primary visual cortex (V1) as early as 40 to $60 \mathrm{~ms}$ upon the appearance of musical notes (Wong et al., 2014). Intriguingly, this early visual cortex engagement was observed only when participants expected 
musical notation to appear consecutively, suggesting the involvement of both feedforward stimulus processing locally in $\mathrm{V} 1$ and the top-down tuning of the visual processing network. The current findings, together with the general observation that perceptual learning with simple features often engages early visual cortex (Furmanski, Schluppeck, \& Engel, 2004; Pourtois, Rauss, Vuilleumier, \& Schwartz, 2008; Sigman et al., 2005), suggest that experts learn to temporarily tune up V1 to be more sensitive to line junctions of musical notation. Future studies should directly test whether line junctions are the critical features that help explain the recruitment of V1 in music reading expertise. In addition, in more complicated contexts, such as those involving accidental signs (the sharps, flats, or natural signs) and the key signature, pitch-related features other than the line junctions may play a role. The extraction of rhythmic information may involve yet another set of features. Future work may consider note sequences in these complicated real-world contexts, and investigate the way various features are combined for a coherent and fluent perception of musical sequences.

Acknowledgements The authors declare no conflict of interest. We thank Gabriel Chan Pak Hong for his help in data collection.

\section{References}

Biederman, I. (1987). Recognition-by-components: A theory of human image understanding. Psychological Review, 94, 115-147.

Brainard, D. H. (1997). The Psychophysics Toolbox. Spatial Vision, 10, 433-436.

Cohen, L., Dehaene, S., Naccache, L., Lehericy, S., Dehaene-Lambertz, G., Henaff, M. A., \& Michel, F. (2000). The visual word form area: Spatial and temporal characterization of an initial stage of reading in normal subjects and posterior split-brain patients. Brain, 123(Pt. 2), 291-307.

Downing, P. E., Jiang, Y., Shuman, M., \& Kanwisher, N. (2001). A cortical area selective for visual processing of the human body. Science, 293(5539), 2470-2473.

Epshtein, B., \& Ullman, S. (2006). Satellite features for the classification of visually similar classes. Proceedings of the IEEE Computer Society Conference on Computer Vision and Pattern Recognition, 2, 2079-2086.

Epstein, R., \& Kanwisher, N. (1998). A cortical representation of the local visual environment. Nature, 392(6676), 598-601.

Fiset, D., Blais, C., Arguin, M., Tadros, K., Ethier-Majcher, C., Bub, D., \& Gosselin, F. (2009). The spatio-temporal dynamics of visual letter recognition. Cognitive Neuropsychology, 26(1), 23-35.

Fiset, D., Blais, C., Éthier-Majcher, C., Arguin, M., Bub, D., \& Gosselin, F. (2008). Features for uppercase and lowercase letter identification. Psychological Science, 19(11), 1161-1168.

Furmanski, C. S., Schluppeck, D., \& Engel, S. A. (2004). Learning strengthens the response of primary visual cortex to simple patterns. Current Biology, 14, 573-578.

García-Pérez, M. A. (1998). Forced-choice staircases with fixed step sizes: Asymptotic and small-sample properties. Vision Research, $38,1861-1881$
Gauthier, I., Wong, A. C.-N., Hayward, W. G., \& Cheung, O. S. (2006). Font tuning associated with expertise in letter perception. Perception, 35, 541-559.

Gibson, B. M., Lazareva, O. F., Gosselin, F., Schyns, P. G., \& Wasserman, E. A. (2007). Nonaccidental properties underlie shape recognition in mammalian and nonmammalian vision. Current Biology, 17(4), 336-340.

Hannagan, T., \& Grainger, J. (2013). Learning diagnostic features: The delta rule does Bubbles. Journal of Vision, 13(8), 17. doi:https://doi. org/10.1167/13.8.17

James, K. H., \& James, T. W. (2005). Letter processing in the visual system: Different activation patterns for single letters and strings, 5(4), 452-466.

Kanwisher, N., McDermott, J., \& Chun, M. M. (1997). The fusiform face area: A module in human extrastriate cortex specialized for face perception. Journal of Neuroscience, 17, 4302-4311.

Lanthier, S. N., Risko, E. F., Stolz, J. A., \& Besner, D. (2009). Not all visual features are created equal: Early processing in letter and word recognition. Psychonomic Bulletin \& Review, 16(1), 67-73.

Maurer, D., Le Grand, R., \& Mondloch, C. J. (2002). The many faces of configural processing. Trends in Cognitive Sciences, 6(6), 255-260.

Mccleery, J. P., Zhang, L., Ge, L., Wang, Z., Christiansen, E. M., Lee, K., \& Cottrell, G. W. (2008). The roles of visual expertise and visual input in the face inversion effect: Behavioral and neurocomputational evidence. Vision Research, 48, 703-715.

McClelland, J. L., \& Rumelhart, D. E. (1981). An interactive activation model of context effects in letter perception. Psychological Review, $88,275-407$

Pelli, D. G. (1997). The videotoolbox software for visual psychophysics: Transforming numbers into movies. Spatial Vision, 10, 437-442.

Pelli, D. G., Burns, C. W., Farell, B., \& Moore-Page, D. C. (2006). Feature detection and letter identification. Vision Research, 46(28), 4646-4674.

Pourtois, G., Rauss, K. S., Vuilleumier, P., \& Schwartz, S. (2008). Effects of perceptual learning on primary visual cortex activity in humans. Vision Research, 48(1), 55-62.

Riesenhuber, M., \& Poggio, T. (1999). Hierarchical models of object recognition in cortex. Nature Neuroscience, 2(11), 1019-1025.

Sigman, M., Pan, H., Yang, Y., Stern, E., Sillbersweig, D., \& Gilbert, C. D. (2005). Top-down reorganization of activity in the visual pathway after learning a shape identification task. Neuron, 46, 823-835.

Szwed, M., Cohen, L., Qiao, E., \& Dehaene, S. (2009). The role of invariant line junctions in object and visual word recognition. Vision Research, 49(7), 718-725.

Szwed, M., Dehaene, S., Kleinschmidt, A., Eger, E., Valabrègue, R., Amadon, A., \& Cohen, L. (2011). Specialization for written words over objects in the visual cortex. NeuroImage, 56(1), 330-344.

Testolin, A., Stoianov, I., \& Zorzi, M. (2017). Letter perception emerges from unsupervised deep learning and recycling of natural image features. Nature Human Behaviour, 1(9), 657.

Tong, M. H., Joyce, C. A., \& Cottrell, G. W. (2008). Why is the fusiform face area recruited for novel categories of expertise? A neurocomputational investigation. Brain Research, 1202, 14-24.

Ullman, S. (2007). Object recognition and segmentation by a fragmentbased hierarchy. Trends in Cognitive Science, 11(2), 58-64.

Wong, Y. K., \& Gauthier, I. (2010a). A multimodal neural network recruited by expertise with musical notation. Journal of Cognitive Neuroscience, 22(4), 695-713.

Wong, Y. K., \& Gauthier, I. (2010b). Holistic processing of musical notation: Dissociating failures of selective attention in experts and novices. Cognitive, Affective \& Behavioral Neuroscience, 10, 541551.

Wong, Y. K., \& Gauthier, I. (2012). Music-reading expertise alters visual spatial resolution for musical notation. Psychonomic Bulletin \& Review, 19, 594-600. 
Wong, Y. K., Peng, C., Fratus, K. N., Woodman, G. F., \& Gauthier, I. (2014). Perceptual expertise and top-down expectation of musical notation engages the primary visual cortex. Journal of Cognitive Neuroscience, 26(8), 1629-1643. doi:https://doi.org/10.1162/jocn_ a 00616

Wong, Y. K., Twedt, E., Sheinberg, D., \& Gauthier, I. (2010). Does Thompson's Thatcher effect reflect a face-specific mechanism? Perception, 39, 1125-1141.
Wong, Y. K., \& Wong, A. C.-N. (2016). Music-reading training alleviates crowding with musical notation. Journal of Vision, 16(8), 15. doi: https://doi.org/10.1167/16.8.15

Yin, R. K. (1969). Looking at upside-down faces. Journal of Experimental Psychology, 81(1), 141-145. 